\title{
A implementação da avaliação formativa e sumativa no ensino da educação física
}

\author{
Francisco Miranda Gonçalves \\ Escola Superior de Educação do Instituto Politécnico de Viana do Castelo - ESE-IPVC, Portugal \\ Centro de Estudos em Educação, Tecnologias e Saúde - CI\&DETS, Portugal
}

Ricardo Franco Lima Escola Superior de Desporto e Lazer do Instituto Politécnico de Viana do Castelo - ESDL-IPVC, Portugal

\begin{abstract}
RESUMO
A avaliação consiste na recolha de informações necessárias para um melhor desempenho do processo de aprendizagem dos alunos. O presente estudo teve como objetivos: i) conhecer até que ponto os professores do ensino secundário recorrem à avaliação formativa; ii) perceber a importância que os professores de Educação Física atribuem à avaliação formativa. Fizeram parte do estudo 71 professores dos ensinos básico e secundário regular, assim como 850 alunos, sendo 291 do $3^{\circ}$ ciclo, 559 do ensino secundário. De acordo com os resultados obtidos, concluiu-se que se recorre à avaliação formativa nos processos de ensino e que se atribui grande importância à mesma por parte dos professores. Verificou-se que classificação da Avaliação Sumativa tende a aumentar, relativamente à Avaliação Formativa.
\end{abstract}

PALAVRAS-CHAVE: Educação Física. Avaliação. Aprendizagens. Professores. Alunos.

\section{THE IMPLEMENTATION OF FORMATIVE AND SUMMATIVE EVALUATION IN THE TEACHING OF PHYSICAL EDUCATION}

\begin{abstract}
The evaluation consists of collecting information necessary for a better performance of the students' learning process. The present study had the following objectives: i) to know the extent to which teachers of secondary education resort to formative evaluation; ii) perceive the importance that physical education teachers attribute to formative evaluation. The study included 71 teachers of basic and secondary education, as well as 850 students, 291 of the third cycle, 559 of secondary education. According to the results, it was concluded that formative evaluation in teaching processes is used and that it is given great importance to the same by teachers. It was verified that the classification of the Summative Evaluation tends to increase, relative to the Formative Evaluation.
\end{abstract}

KEYWORDS: Physical Education. Evaluation. Learning. Teachers. Students. 


\section{LA IMPLEMENTACIÓN DE LA EVALUACIÓN FORMATIVA Y SUMATIVA EN LA ENSEÑANZA DE LA EDUCACIÓN FÍSICA}

\section{RESUMEN}

La evaluación consiste en la recogida de información necesaria para un mejor desempeño del proceso de aprendizaje de los alumnos. El presente estudio tuvo como objetivos: i) conocer hasta qué punto los profesores de enseñanza secundaria recurren a la evaluación formativa; ii) percibir la importancia que los profesores de Educación Física atribuyen a la evaluación formativa. Se realizaron parte del estudio 71 profesores de las enseñanzas básica y secundaria regular, así como 850 alumnos, siendo 291 del $3^{\circ}$ ciclo, 559 de la enseñanza secundaria. De acuerdo con los resultados obtenidos, se concluyó que se recurre a la evaluación formativa en los procesos de enseñanza y que se atribuye gran importancia a la misma por parte de los profesores. Se verificó que la clasificación de la Evaluación Sumativa tiende a aumentar, en relación a la Evaluación Formativa.

PALABRAS ClAVE: Educación Física. Evaluación. Aprendizaje. Profesores. Estudiantes.

\section{INTRODUÇÃO}

Avaliar em educação pressupõe a obtenção de informações relativas à qualidade e à eficiência de uma determinada ação educativa e permite emitir um juízo de valor a seu respeito. Caracteriza-se também pela utilização do juízo emitido, permitindo decisões que implicam ações posteriores (DAMON, 2007).

A avaliação ajuda o professor a refletir sobre as suas práticas, estratégias e adequação das mesmas aos alunos e ajuda os mesmos a aprender, acompanhando todo o seu envolvimento nas tarefas didáticas (SAUNDERS, 2010).

Professores e alunos devem ter a noção de que a avaliação consiste na recolha de informação necessária para melhorar o desempenho (GONÇALVES, 2012). A avaliação é fundamental ao longo de todo o processo de ensino aprendizagem, cujo objetivo é a superação das dificuldades dos alunos, sem perder de vista o patamar do sucesso. É um processo que se desenvolve em diferentes momentos, com diferentes objetivos, sendo por isso constituída pelas avaliações diagnóstica, formativa e sumativa, que têm, respetivamente, a finalidade de: verificar conhecimento e habilidades previstas, a fim de orientar o aluno para novas aprendizagens; informar sobre as aprendizagens possibilitando reajustes no processo e superação das dificuldades; classificar, e/ou realizar um balanço, de acordo com os objetivos previstos (BLANCHARD, 2003). Neste sentido realça-se a complementaridade e articulação das diferentes modalidades de avaliação (MORAIS; NEVES; AFONSO, 2005).

Em termos de modalidades e momentos de avaliação, enquanto que a avaliação sumativa é efetuada no final de um período de ensino para decidir a continuação de um 
determinado programa, a avaliação formativa é realizada durante o decurso do processo de ensino e de aprendizagem, no sentido do seu aperfeiçoamento (HUMPHRIES et al., 2012).

O interesse que a avaliação formativa tem vindo a alcançar, em todos os seus contextos, pressupõe a necessidade de se aumentar, de forma significativa, a importância para o processo de ensino aprendizagem dos alunos, como, também, perceber se os professores a aplicam adequadamente (LEACH; MOON, 2000).

A avaliação sumativa, ou final, tem mais por função constatar o insucesso do que promover o sucesso escolar (ATKINS; KELLY; MORRISON, 2001). Enquanto que à avaliação sumativa se costumam atribuir as funções sociais da avaliação (seleção, hierarquização, certificação), à avaliação formativa atribuem-se as funções pedagógicas (ajuda, diagnóstico, regulação...). Assim, a avaliação sumativa deverá ser entendida como um meio para se conhecer mais sobre uma determinada realidade, numa perspetiva de se aperfeiçoarem processos futuros (MORAIS; NEVES; AFONSO, 2005).

Hoje, cabe aos professores a realização de uma avaliação que tenha em vista a especificidade dos alunos, pedagogia diferenciada, onde se avaliem os diferentes domínios, que se apoiem os alunos com dificuldades, e que se modifiquem práticas pedagógicas e de avaliação (ALEXANDER, 2001).

A avaliação formativa é a única que permite promover uma aprendizagem mais autónoma por parte do aluno, auxiliando também o professor na construção de uma avaliação mais justa e eficiente (GONÇALVES, 2012). A avaliação formativa preocupa-se em determinar o grau de domínio de uma determinada tarefa de aprendizagem e indicar a parte não dominada. Desta forma, o objetivo não é atribuir uma nota ou um certificado ao aluno, é ajudar tanto o aluno como o professor a deterem-se na aprendizagem específica necessária ao domínio da matéria (WESTON, 2004). Esta ajuda o aluno a aprender e o professor a ensinar, funcionando como um duplo feedback (HUMPHRIES et al., 2012).

A avaliação formativa deve estar, sobretudo, centrada no aluno e preocupada com os objetivos a alcançar (CHEN et al., 2012). Por sua vez, o processo formativo é mais complexo e, num certo sentido, mais sofisticado, ou mais rico, do ponto de vista teórico (BROWN, 2004). Trata-se de uma avaliação interativa, centrada nos processos cognitivos dos alunos e associada aos processos de feedback, de regulação, de auto-avaliação e de regulação das aprendizagens (CLARK, 2002).

O processo de avaliação formativa, será bastante benéfico para os alunos, daí a necessidade de os professores dominarem a avaliação formativa, pois é esta que deve acompanhar todo o processo ensino aprendizagem identificando aprendizagens bem-sucedidas 
e as que levantam dificuldades, para que possam ser ultrapassadas levando os alunos à proficiência e ao sucesso. Por sua vez, a avaliação formativa é interna ao processo, é contínua, analítica e mais centrada sobre o aprendente do que sobre o produto acabado (DANUSSO; TESTA; VICENTINI, 2010).

Em termos do processo de ensino e de aprendizagem, inicialmente o professor recolhe informações relativas aos progressos e dificuldades dos alunos, posteriormente interpreta a informações diagnosticando os fatores que lhes dão origem, e por fim, tenta adaptar as atividades de ensino de acordo as interpretações, respondendo adequadamente a cada situação específica (BROWN, 2004). A orientação individualizada ao longo do processo é mais vantajosa do que uma remediação a posteriori (HALL; SMITH, 2006). De acordo com esta premissa, quem avalia tem de dar a conhecer ao objeto de avaliação a forma como vai ser avaliado, e ainda, quais os parâmetros e critérios de avaliação que vão legitimar o seu desempenho (DARABI, 2005). As informações recolhidas pela avaliação devem ser transmitidas a alunos e encarregados de educação para que, em conjunto, se possa aperfeiçoar todo esse processo.

Importa ainda salientar a auto avaliação como aspeto fundamental a ter em conta, pois é uma das pedras basilares da avaliação formativa (RASMUSSEN; FRICHE, 2011). Permite ao aluno a identificação dos erros de percurso, consciencializando-o da sua situação, e contribuindo, desta forma para a melhoria do seu desempenho (GIBBONS, 2003).

A avaliação formativa é um processo determinante na melhoria dos resultados dos alunos através da utilização de tarefas e que é uma avaliação orientada para as aprendizagens, porque tem um papel muito significativo nas formas como os alunos se preparam e organizam ativamente para aprender melhor (ROTHSTEIN; JACOBSEN, 2006). Porém, é uma modalidade de avaliação ainda pouco praticada, em parte porque as práticas de avaliação são basicamente dominadas pela avaliação sumativa e também devido à necessidade de ser realizada em todos os momentos, aulas (GONÇALVES, 2012). Tem como principal função o acompanhamento do aluno, permitindo, assim, verificar os comportamentos demonstrados perante cada situação do processo ensino aprendizagem. É através da avaliação formativa que, posteriormente se devem retirar as informações necessárias a classificação do aluno (STUFFLEBEAM; WINGATE, 2005). Os alunos devem ser avaliados ao longo das aulas, com frequência, à medida que vão fazendo os exercícios, sem nervosismo e ansiedade (GIAMBONA; VASSALLO; VASSILIADIS, 2011).

A avaliação formativa requer novas práticas pedagógicas e, portanto, exige alterações significativas no contexto educativo. Os alunos devem participar ativamente na sua 
aprendizagem, para a avaliação funcionar de uma forma formativa, os resultados devem ser utilizados para modificar o ensino e a aprendizagem e por fim a avaliação tem o potencial de afetar não apenas a aprendizagem do aluno, mas também a motivação e a auto-estima (ATKINS; KELLY; MORRISON, 2001; MARTIN, 2002).

\section{METODOLOGIA}

Fizeram parte do estudo 71 professores dos ensinos básico e secundário regular, assim como 850 alunos, sendo 291 do $3^{\circ}$ ciclo e 559 do ensino secundário. Para se efetuar a comparação da classificação da avaliação formativa com a da avaliação sumativa, no domínio psicomotor, foram ainda analisadas 60 unidades didáticas ( 17 do $3^{\circ}$ ciclo e 43 do ensino secundário).

Com o objetivo de avaliar as práticas de avaliação formativa, e a importância atribuída à mesma, foi elaborado um questionário, que consistiu no preenchimento individual, anónimo e confidencial, constituído por quatro grupos: o primeiro pretendeu caracterizar o praticante, pelo género, e disciplina que leciona; o segundo constituído por duas questões de resposta de escolha múltipla; o terceiro relativo à prática da avaliação formativa, constituído por 18 itens e o quarto relativo à importância da avaliação formativa, constituído por 22 itens (segundo uma escala de Likert, pontuados de 1 a 5, sendo 1-nada importante e 5-muito importante). O score máximo para a prática da avaliação formativa foi de 105 pontos e para a importância da avaliação formativa foi de 90 pontos. Estes scores foram transformados em percentagem assumindo $100 \%$ o score máximo possível para cada uma das variáveis. As diferentes disciplinas foram agrupadas em áreas, nomeadamente Letras e Humanidades (Inglês (8), História (2), Espanhol (1), Filosofia (4), Português (7), Ciências (Biologia/Geologia) (6), Físico-Química (7), Geografia (2), Matemática (9), Geometria (1) e Economia (1), e Educação Física (10).

O questionário foi elaborado especificamente para o presente estudo, e o tratamento de dados foi realizado através do programa SPSS - Statistical Package for the Social Sciences 20.0. Foi efetuada a estatística descritiva e realizada uma ONE-WAY ANOVA, considerando as variáveis dependentes importância da avaliação formativa e a prática da avaliação formativa nas respostas às questões dos grupos 2, 3 e 4 do questionário. Relativamente aos grupos 3 e 4 , a análise foi realizada considerando o fator fixo áreas com 3 níveis e o teste Post Hoc de Bonferroni para diferenças entre níveis. Foi considerado o valor de $\alpha=0.05$.

Em relação à opinião de cada docente, foi enviada uma tabela em formato Excel, onde foi efetuada a caracterização dos participantes, designação da Unidade Didática e classificação 
de cada aluno. A classificação dos alunos foi efetuada numa escala de 0-100\%.

\section{RESULTADOS}

Acerca da capacidade do docente classificar os alunos sem o momento de avaliação sumativa, 38 docentes responderam afirmativamente, e 21 responderam negativamente. Já no que concerne à formação inicial que o professor teve o habilitar de forma a ser capaz de responder às necessidades dos alunos, 9 docentes responderam negativamente, e 50 afirmativamente.

No que se prende às práticas de avaliação formativa e à importância atribuída à mesma modalidade de avaliação, os resultados obtidos foram de uma média e desvio padrão de: prática

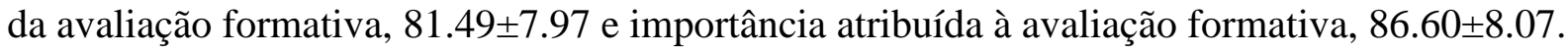
Relativamente à avaliação formativa e à importância atribuída à avaliação formativa, os resultados obtidos, de acordo com as áreas foram:

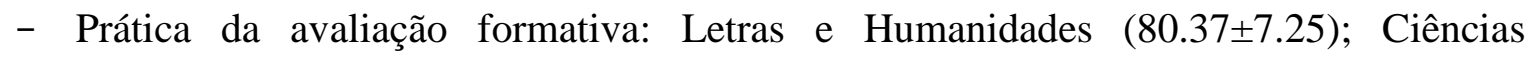

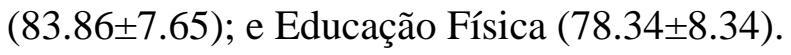

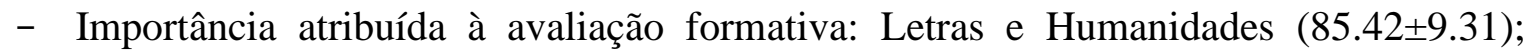

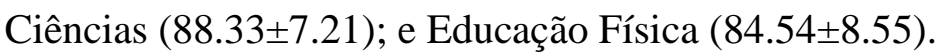

Foram ainda efetuadas comparações entre a prática de avaliação formativa e a importância da avaliação formativa em várias dimensões de análise, como se apresenta na tabela 1.

Tabela 1. Resultados das comparações entre a prática da avaliação formativa e importância da avaliação formativa com as questões de capacidade de classificar os alunos com a avaliação formativa, formação dos professores ajuda a dar resposta às necessidades, e diferentes áreas.

\begin{tabular}{cccc} 
& Relação & F & Sig \\
\hline $\begin{array}{c}\text { Prática da } \\
\text { avaliação } \\
\text { formativa }\end{array}$ & Capacidade de classificar os alunos com a avaliação \\
formativa & 2.580 & 0.114 \\
\hline $\begin{array}{c}\text { Importância da } \\
\text { avaliação } \\
\text { formativa }\end{array}$ & $\begin{array}{c}\text { Formação dos professores ajuda a dar resposta às } \\
\text { necessidades } \\
\text { Áreas }\end{array}$ & 0.481 & 0.491 \\
\hline $\begin{array}{c}\text { Cormação dos professores ajuda a dar resposta às } \\
\text { focessidades } \\
\text { formativa }\end{array}$ & 1.868 & 0.164 \\
\hline
\end{tabular}

Foi considerado o valor de $\alpha=0.05$. 
Os valores da prática de avaliação formativa e da importância da avaliação formativa são independentes das respostas obtidas relativamente às questões da capacidade de classificar os alunos com a avaliação formativa, da formação dos professores ajudar a dar resposta às necessidades e às diferentes áreas.

No que diz respeito à comparação da avaliação formativa com a avaliação sumativa verificou-se que o número total de aulas da Unidade Didática foi de $76.7 \pm 15.0$ para a avaliação formativa e de $79.5 \pm 13.6$ para a avaliação sumativa. Subdividindo por ciclo de estudos, para a avaliação formativa, verificaram-se valores de 73.6 \pm 17.0 para o $3^{\circ}$ ciclo e de $78.3 \pm 13.6$ para o ensino secundário. Relativamente à avaliação sumativa, os valores passam $75.8 \pm 15.9$ e $81.3 \pm 11.9$, respetivamente.

\section{DISCUSSÃO}

Através dos resultados apresentados verifica-se que os participantes evidenciam elevado enfase atribuído à avaliação formativa, prática de avaliação formativa=81.49 \pm 7.97 e importância da avaliação formativa=86.60 \pm 8.07 . Observa-se que foram os docentes da área das ciências que alcançaram valores das médias mais elevados relativamente à prática e importância da avaliação formativa, valores de $83.86 \pm 7.65$; e $88.33 \pm 7.21$, respetivamente. Em relação às outras áreas disciplinares analisadas os valores foram de: Letras e Humanidades, 80.37 \pm 7.25 ;

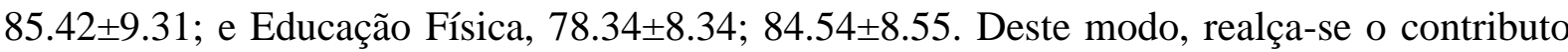
dos docentes, para a melhoria das aprendizagens, da motivação e auto-estima, numa perspetiva de progressão, como demonstrado em estudos sobre a temática (ATKINS; KELLY; MORRISON, 2001; MARTIN, 2002).

Apesar da elevada importância que atribuem à referida modalidade de avaliação, observa-se que 21 professores afirmaram não serem capazes de classificar sem o momento da avaliação sumativa, enquanto que 38 afirmaram o contrário. A bibliografia defende que enquanto um professor não se conceber como alguém capaz de fazer o aluno aprender, não tem razão alguma para se interessar pela avaliação formativa (HUMPHRIES et al., 2012; ROTHSTEIN; JACOBSEN, 2006). Enquanto um professor julgar que o fracasso é normal, que há bons e maus alunos, que seu trabalho é o de dar aulas e não o de assegurar uma regulação individualizada dos processos de aprendizagem, a avaliação formativa continuará a ser indiferente para ele (GIAMBONA; VASSALLO; VASSILIADIS, 2011; GONÇALVES, 2012).

No entanto, o facto de não se sentirem capazes, pode ser explicado pelo conforto em objetivar a avaliação, à insegurança na fundamentação da sua avaliação ou à facilidade de 
comunicação de um número (BLANCHARD, 2003; DAMON, 2007). Os problemas revelados pelos professores sobre a avaliação surgem associados à falta de uniformização dos critérios de avaliação, aos processos e componentes da avaliação e à dificuldade em construir instrumentos adequados (DARABI, 2005; LEACH; MOON, 2000; MORAIS; NEVES; AFONSO, 2005).

De acordo com os resultados obtidos, verifica-se que há mais professores, nas áreas das Letras e Humanidades (dos quais 15 responderam afirmativamente, e 7 negativamente) e de Educação Física (10 e 1 respetivamente), que dizem ser capazes de avaliar sem o momento de avaliação sumativa, comparativamente com professores na área das Ciências (dos quais 13 responderam afirmativamente, e 13 negativamente). Para empenhar os professores no processo de mudança eles precisam de novos conhecimentos (DANUSSO; TESTA; VICENTINI, 2010). Torna-se evidente que a formação contínua deve ser encarada como um modo de promover o desenvolvimento profissional, para colmatar carências na formação inicial do professor (STUFFLEBEAM; WINGATE, 2005).

No entanto, o facto de serem ou não capazes de classificar sem o momento da avaliação sumativa, não se relaciona com a prática de avaliação formativa nem com a importância da avaliação formativa, pois $p>0.05$. Além disso, verifica-se que os professores da área das ciências, que em maior número referiram não ser capazes de classificar sem o momento de avaliação sumativa, foram os que alcançaram posteriormente as maiores médias relativamente à prática de avaliação formativa e à importância da avaliação formativa. Estes resultados foram obtidos independentemente de terem respondido afirmativa ou negativamente às questões sobre a capacidade de classificar os alunos com a avaliação formativa e sobre a formação de que foram alvo para os habilitar a dar respostas às necessidades dos alunos, à imagem do que postulam os estudos realizados a nível Europeu (ALEXANDER, 2001; CHEN et al., 2012; GIAMBONA; VASSALLO; VASSILIADIS, 2011; ROTHSTEIN; JACOBSEN, 2006).

Os resultados evidenciam a importância atribuída à avaliação formativa, pois só através dela, é possível detectar as dificuldades dos alunos e, consequentemente, arranjar as melhores opções para ultrapassar essas dificuldades e seguidamente, atingir os melhores resultados (HUMPHRIES ET AL, 2012; SAUNDERS, 2010). No entanto, como se pode verificar pelos resultados apresentados, a classificação dos alunos, no domínio psicomotor, é superior quando se opta pela avaliação formativa comparativamente à sumativa. Assim, de acordo com os resultados obtidos, os docentes ainda necessitam de recorrer à aula relativa à avaliação sumativa para classificarem os alunos. Desta feita, atribuem pouca importância à avaliação formativa. Pode ainda verificar-se que a diferença entre a avaliação formativa e sumativa é menor no $3^{\circ}$ ciclo, 2 pontos percentuais, do que no ensino secundário, sendo neste ciclo de 3 pontos. 
Os resultados demostram uma preocupação no que diz respeito à intenção de se trabalhar no sentido do sucesso e da proficiência, onde a avaliação formativa tem um papel fundamental, salientando-se a necessidade na manutenção das práticas formativas, na alteração de práticas educativas, bem como a formação contínua de professores (BLANCHARD, 2003; BROWN, 2004; DANUSSO; TESTA; VICENTINI, 2010).

\section{CONCLUSÕES}

De acordo com os resultados obtidos conclui-se que o conceito de avaliação formativa assume lugar de destaque no processo de ensino e de aprendizagem, ao qual se atribui grande importância por parte do corpo docente. Os resultados evidenciaram a necessidade de formação contínua por parte dos docentes a este nível, pois denotou-se uma dificuldade de operacionalização da classificação final dos alunos sem a avaliação sumativa. Há, então, a necessidade de se alterar esta prática para se responder às necessidades dos alunos e para que a escola consiga alcançar, ainda melhores resultados, caminhando no sentido da excelência.

O estudo realizado permitiu ainda constatar que a classificação da avaliação formativa é significativamente diferente da classificação proveniente da avaliação sumativa, pois esta última tende a ser superior relativamente à classificação da avaliação formativa. No $3^{\circ}$ ciclo a classificação da avaliação formativa está mais próxima da avaliação sumativa, comparativamente ao ensino secundário.

Embora sendo dada bastante importância à avaliação formativa, alguns professores afirmaram a incapacidade de classificarem os seus alunos sem o momento de avaliação sumativa.

No entanto, o facto de não se sentirem competentes a este nível, pode ser explicado pelo conforto em objetivar a avaliação, à insegurança na fundamentação da sua avaliação ou à facilidade de comunicação de um número. Parece claro que a formação contínua contribui para o desenvolvimento profissional do docente.

Os resultados demostram uma intenção respeitante ao objetivo de se trabalhar no sentido do sucesso e da proficiência, onde a avaliação formativa tem um papel fundamental, salientando-se a necessidade na manutenção das práticas formativas, na alteração de práticas educativas, bem como a formação contínua de professores.

\section{REFERÊNCIAS}


ALEXANDER, R. Culture and pedagogy: International comparisons in Primary Education. Journal of Education for Teaching, v. 27, n. 3, p. 281-289, 2001.

ATKINS, D. H., KELLY, K. T., \& MORRISON, G. S. Development of the child evaluation measure: An assessment of children's learning across disciplines and in multiple contexts. Educational \& Psychological Measurement, v. 61, n. 3, p. 505, 2001.

BLANCHARD, J. Targets, assessment for learning, and whole-school improvement. Cambridge Journal of Education, v. 33, n. 2, p. 257, 2003.

BROWN, N. What makes a good educator? The relevance of meta programmes. Assessment \& Evaluation in Higher Education, v. 29, n. 5, p. 515-533, 2004.

CHEN, W., MASON, S., STANISZEWSKI, C., UPTON, A., \& VALLEY, M. Assessing the quality of teachers' teaching practices. Educational Assessment, Evaluation and Accountability, v. 24, n. 1, p. 25-41, 2012.

CLARK, C. Effective multicultural curriculum transformation across disciplines. Multicultural Perspectives, v. 4, n. 3, p. 37-46, 2002.

DAMON, W. Dispositions and teacher assessment: The need for a more rigorous definition. Journal of Teacher Education, v. 58, n. 5, p. 365-369, 2007.

DANUSSO, L., TESTA, I., \& VICENTINI, M. Improving prospective teachers' knowledge about scientific models and modelling: Design and evaluation of a teacher education intervention. International Journal of Science Education, v. 32, n. 7, p. 871-905, 2010.

DARABI, A. A. Application of cognitive apprenticeship model to a graduate course in performance systems analysis: A case study. Educational Technology Research and Development, v. 53, n. 1, p. 49-64, 2005.

GIAMBONA, F., VASSALLO, E., \& VASSILIADIS, E. Educational systems efficiency in European Union Countries. Studies in Educational Evaluation, v. 37, n. 2-3, p. 108-122, 2011.

GIBBONS, B. A. Supporting elementary science education for english learners: A constructivist evaluation instrument. Journal of Educational Research, v. 96, n. 6, p. 371-380, 2003.

GONÇALVES, F. Formative evaluation in Physical Education initial teacher training courses. Journal of Physical Education and Sport Management, v. 3, n. 1, p. 1-5, 2012.

HALL, T. J., \& SMITH, M. A. Teacher planning, instruction and reflection: what we know about teacher cognitive processes. Quest, v. 58, n. 4, p. 424-442, 2006.

HUMPHRIES, C. A., HEBERT, E., DAIGLE, K., \& MARTIN, J. Development of a Physical Education teaching efficacy scale. Measurement, Physical Education \& Exercise Science, v. 16, n. 4, p. 284-299, 2012.

LEACH, J., \& MOON, B. Pedagogy, information and communications technology and teachers' professional knowledge. Curriculum Journal, v. 11, n. 3, p. 385-404, 2000. 
MARTIN, K. A. Development and validation of the coaching staff cohesion scale. Measurement. Physical Education \& Exercise Science, v. 6, n. 1, p. 23-42, 2002.

MORAIS, A. M., NEVES, I. P., \& AFONSO, M. Teacher training processes and teachers' competence-a sociological study in the primary school. Teaching and Teacher Education: An International Journal of Research and Studies, v. 21, n. 4, p. 415-437, 2005.

RASMUSSEN, A.; FRICHE, N. Roles of assessment in secondary education: Participant perspectives. Educational Assessment, Evaluation and Accountability, v. 23, n. 2, p. 113-129, 2011.

ROTHSTEIN, R.; JACOBSEN, R. The goals of education. Phi Delta Kappan, v. 88, n. 4, p. 264-272, 2006.

SAUNDERS, L. The challenges of small-scale evaluation in a foreign country: Reflections on practice. Educational Assessment, Evaluation and Accountability, v. 22, n. 3, p. 199-213, 2010.

STUFFLEBEAM, D. L.; WINGATE, L. A. A self-assessment procedure for use in evaluation training. American Journal of Evaluation, v. 26, n. 4, p. 544-561, 2005.

WESTON, T. Formative evaluation for implementation: Evaluating educational technology applications and lessons. American Journal of Evaluation, v. 25, n. 1, p. 51-64, 2004.

\section{SOBRE OS AUTORES}

Francisco Miranda Gonçalves é Doutor em Ciências da Educação Física e Desporto pela Universidade de Trás-os-Montes e Alto Douro - Portugal, e especialista em intervenção didática e avaliação. É professor supervisor no Instituto Politécnico de Viana do Castelo e pesquisador do Centro de Estudos em Educação, Tecnologias e Saúde (CI\&DETS), Portugal.

E-mail: franciscojmg@gmail.com

Ricardo Franco Lima é Doutor em Ciências do Desporto pela Universidade de Trás-os-Montes e Alto Douro - Portugal, e especialista em educação física escolar. É professor da Escola Superior de Desporto e Lazer do Instituto Politécnico de Viana do Castelo (ESDL-IPVC), Portugal.

E-mail: ricardofrancolima@hotmail.com 